THEORY AND METHODS

\title{
An alternative approach to quantifying and addressing inequity in healthcare provision: access to surgery for lung cancer in the east of England
}

\author{
John Battersby, Julian Flowers, Ian Harvey
}

J Epidemiol Community Health 2004;58:623-625. doi: 10.1136/jech.2003.013391

See end of article for authors' affiliations

Correspondence to: Dr J Battersby, Southern Norfolk Primary Care Trust, St Andrew's House, St Andrew's Business Park, Thorpe St Andrew, Norwich NR7 OHT, UK. johnb@doctors.org.uk

Accepted for publication 6 November 2003

\begin{abstract}
Study objective: Equitable access to healthcare services should be monitored routinely. This study compares provision of surgery for non-small cell lung cancer in the east of England with incidence of nonsmall cell lung cancer. In addition to conventional comparisons, process control charts are used to identify areas in which access seems to be significantly different from average.

Design: Ecological comparison of surgery rates for non-small cell lung cancer between 1998 and 2000 and incidence of non-small cell lung cancer over the same time period.

Setting: Population of Norfolk, Suffolk, Cambridgeshire.

Participants: The denominator was the resident population. Numerators were 4092 deaths from non-small cell lung cancer and 387 surgical procedures for lung cancer.

Main results: Incidence of non-small cell lung cancer by primary care trust (PCT) does not correlate with surgical procedure rate, in men $r=0.37$ (95\% confidence intervals -0.14 to 0.72 ), in women $r=0.07$ (95\% confidence intervals -0.43 to 0.53 ). Control charts indicate that the surgery rate is significantly different from average in three PCTs, high in one and low in two others. The optimum surgery rate is unclear but raising it from $9 \%$ to a theoretical level of $15 \%$ would mean no PCTs have above average rates while six PCTs have rates that are significantly low.

Conclusions: There does not seem to be equity of access to surgery for patients with non-small cell lung cancer in the east of England. Control charts can help both to identify areas where access is particularly high or low, and also to monitor performance against a theoretical optimum surgery rate.
\end{abstract}

$\mathrm{E}$ quitable access to healthcare services is of enormous current concern in health care. The recent English primary care trust (PCT) planning guidance, "Improvement, Expansion and Reform"1 ${ }^{\prime 1}$ identifies the requirement that service planning be informed by equity audit. There is therefore a need for methods of performing equity audit that are effective in highlighting potential areas of inequity and that do not require much in the way of resources. Those carrying out equity audits also need to present the results of audits to policymakers in ways that are easily understood.

Equity audit is described in detail elsewhere. ${ }^{23}$ It entails measuring health need in a defined population, using proxies such as disease incidence or mortality. Need is then compared with a measure of service provision for the same population. Equitable provision implies that populations with higher need make greater use of services than populations with lower levels of need. If inequity is identified, it is necessary to establish where in the care pathway it arises. The audit loop can then be completed by intervening to rectify the inequitable provision and re-auditing.

Lung cancer treatment has not been the subject of equity audit before and yet lung cancer is a significant public health issue. It is the third most common cause of death in the UK and it accounts for almost a quarter of all cancer mortality in England and Wales. The prognosis from lung cancer is poor with overall five year survival rates of around 5\%. ${ }^{4}$ Non-small cell lung cancers (NSCLC) account for around $80 \%$ of lung cancers in the UK and, although evidence is limited, it is generally agreed that surgery provides the only hope of curing NSCLC at the present time. ${ }^{5}$ Some observational studies suggest that in appropriately selected patients, surgery may improve five year survival to around $70 \%$.
Cancer registry data from the UK show lower, though none the less increased, survival rates for NSCLC after surgery. ${ }^{6}$

We have performed a study comparing rates of surgery for NSCLC in eastern England against a proxy measure of need (incidence rate of NSCLC). As well as presenting the results using conventional graphs, we have used process control methods to highlight areas in which provision appears unaccountably low and we have compared the overall provision of surgery with the government recommended levels. ${ }^{7}$

\section{METHOD}

Incidence data were used as a proxy measure of need for surgery. NSCLC age-sex standardised incidence rates for PCTs in Norfolk, Suffolk, and Cambridgeshire were obtained from the East Anglian Cancer Intelligence Unit for the years 1998 to 2000. Numbers of incident cases by sex were also obtained for 1997.

The hospital episode statistics (HES) dataset was used to identify all relevant procedures carried out on patients with lung cancer (ICD 10 code C33 and C34) from 1997 to 2000. The relevant procedures (OPCS codes E44.1, E54. ${ }^{*}$, E57.4 and E57.8) were identified from the lung cancer appendix to the Cancer Data Manual. ${ }^{8}$ These data were used to calculate agesex standardised procedure rates for each PCT. For the calculation of both incidence and procedure rates, participants were assigned to a PCT by their postcode of residence.

Conventional scatter plots of procedures (provision) against incidence (need) were prepared for both men and

Abbreviations: PCT, primary care trust; NSCLC, non-small cell lung cancer; HES, hospital episode statistics 
women. Both incidence and procedure rates were also plotted against a weighted average of the PCT overall score on the index of multiple deprivation (IMD) 2000, ${ }^{9}$ to describe the association between these measures and deprivation.

Process control charts were also produced..$^{10}$ This entailed plotting the square root of the number of procedures against the square root of the number of cases minus the number of procedures for each PCT. Three sigma control limits were superimposed on the plot according to the method described by Mostellor and Tukey, ${ }^{11}$ these reflect the range within which $99 \%$ of data points would be expected to lie in a stable system. A further control chart was produced setting the process mean at $15 \%$. This level was chosen because the English Clinical Outcomes Guidance for lung cancer states that, "As many as $20 \%$ of patients may be suitable for surgery and every effort should be made to identify these individuals."

\section{RESULTS}

There are 17 PCTs in Norfolk, Suffolk, and Cambridgeshire with a total population of 2.18 million residents. Between 1997 and 2000 there were a total of 4092 cases of NSCLC identified from the East Anglian Cancer Registry (standardised incidence was calculated using data from the 3160 cases occurring between 1998 and 2000). In the same period, 387 surgical procedures were performed on patients with a primary diagnosis of lung cancer (HES dataset).

Although crude incidence of NSCLC seemed to correlate with IMD 2000 score (weighted average), age-sex standardised incidence was not correlated with the IMD 2000 score, for men $r=0.48$ (95\% confidence intervals (CI) -0.001 to 0.78 ) and for women $r=0.42$ (95\% CI -0.08 to 0.75$)$. Agesex standardised surgical procedure rates did not correlate with IMD 2000 score either, for men $r=-0.10$ (95\% CI -0.55 to 0.40 ) and for women $r=-0.16$ (95\% CI -0.59 to 0.35 ). The incidence of NSCLC showed no significant correlation with the surgical procedure rate, for men $r=0.37$ (95\% CI -0.14 to 0.72 ) and for women $r=0.07$ (95\% CI -0.43 to $0.53)$. Figure 1 shows the plot for men.

A process control chart was produced using data from 1997 to 2000. Figure 2 shows the process control chart. The chart identifies 3 of 17 PCTs that lie outside the control limits. One PCT lies above the upper control limit and two PCTs lie below the lower control limit. The overall proportion of patients that receive surgery is $9 \%$. Figure 3 shows the same points plotted onto a control chart where the mean and control limits are based on an overall proportion of $15 \%$ of patients receiving surgery. This time six PCTs fall below the lower control limit and there are no PCTs that lie above the upper control limit.

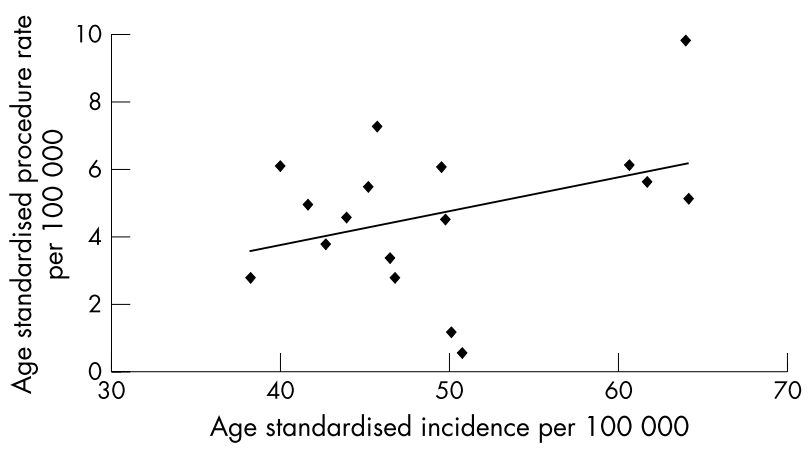

Figure 1 Scatter plot of surgical procedure rate against incidence of non-small cell lung cancer. Data are for men only and each data point represents a primary care trust. Rates are age standardised per 100000 population.

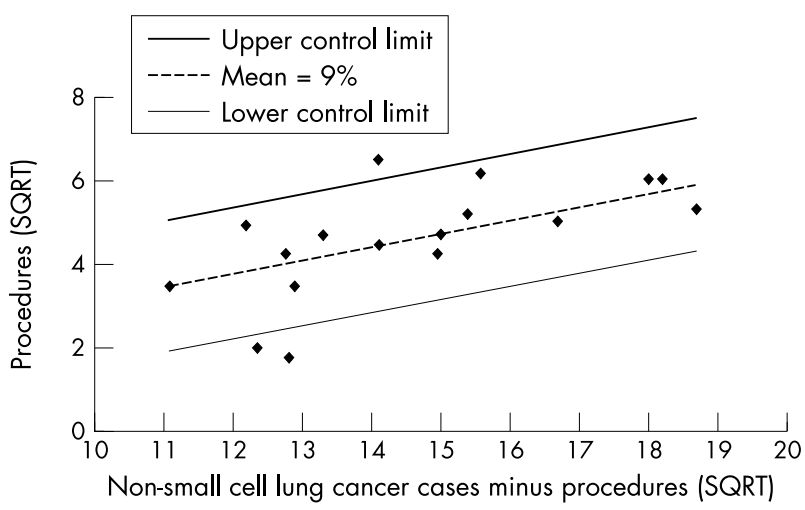

Figure 2 Control chart showing the ratio of cases of non-small cell lung cancer undergoing surgery to cases not undergoing surgery (number of cases minus number of procedures) for all persons. The axes are both on a square root scale and each data point represents a primary care trust (PCT). The control limits are based on the mean for all PCTs in Norfolk, Suffolk, and Cambridgeshire of $9 \%$ cases undergoing surgery.

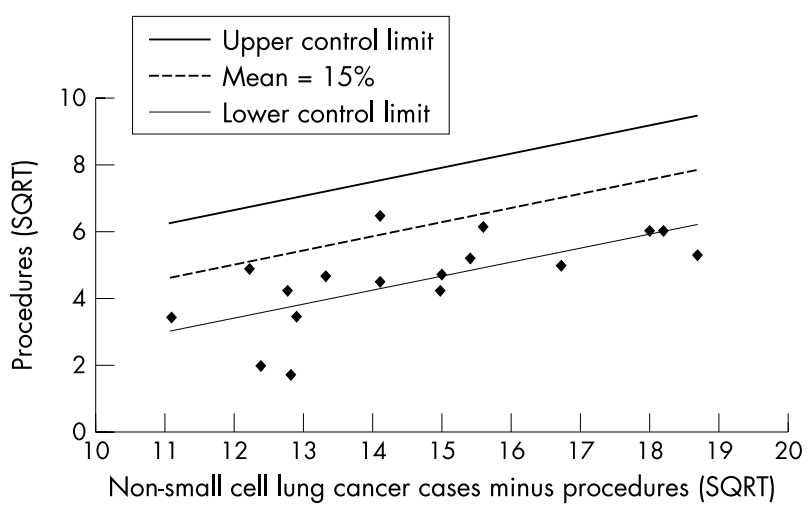

Figure 3 This control chart is identical to the one in figure 2 apart from the control limits, which have been set to assume a mean of $15 \%$ cases undergoing surgery.

\section{DISCUSSION}

A simple scatter plot, as shown in figure 1, together with a correlation coefficient, is a common way of presenting equity data. This alone suggests that provision of lung cancer surgery may not be related to need, as there is no significant relation between incidence and procedure rates. However, the use of a process control chart, often known as a Shewhart chart, permits the identification of PCTs where the variation in case/procedure ratio is greater than would be expected by chance alone. The degree of variation in these PCTs cannot be attributed to inherent variation in measurement and so a specific explanation must be sought. A further advantage of this type of control chart is that denominator data are not required.

A plausible, although unproved, explanation for the PCT that lies above the upper control limit is that the principal provider unit for cardiothoracic surgery in the region lies within the PCT boundary and is, therefore, readily geographically accessible. Patients from the two PCTs below the lower control limit are referred to the same district general hospital. This suggests that the explanation for their position lies in the hospital stage of the care pathway. Further inquiry revealed that until some time after 2000 virtually all lung cancer patients seen at that district general hospital were referred for radiotherapy rather than surgery. This practice appears to be confirmed by the control chart. 
The control chart in figure 3, with a case/procedure rate of $15 \%$, illustrates a further use of control charts in equity audit. As well as identifying which organisations fall outside the calculated control limits, it is possible to make some judgement as to whether the overall provision of services is adequate. If as many as $20 \%$ of patients may be suitable for surgery, a case procedure rate of $9 \%$ could be considered low. Moving the control limits to reflect a case/procedure rate that is closer to the $20 \%$ recommendation shows that 6 of 17 PCTs are no longer achieving case/procedure rates that fall within the control limits. In this situation interventions at a PCT level are unlikely to improve the situation; a whole system approach is required. Of course, the control chart cannot help in deciding whether the $20 \%$ recommendation is appropriate or not.

The method shown does not include any correction for the stage of presentation, as staging data were not available in an appropriate format at the time of the study. However, it seems unlikely that differences in the stage of presentation are a plausible explanation for the outliers in this case, particularly as there does not seem to be any relation between deprivation and surgical procedure rate. Nevertheless, correction for staging would be desirable if the appropriate data were available. It is also possible that the variation in procedure rates between different PCTs could be explained by factors such as variation in patients' views on surgical treatment of lung cancer. However, while patients' views will undoubtedly vary, it seems unlikely that this could account for the geographical variations observed.

Incomplete ascertainment of incident cases is a potential source of bias with this type of study. Cancer registries in the United Kingdom collect data on all incident cancers and the quality of the data that they collect is consistently high. In addition to routine data collection, the East Anglian Cancer Intelligence Unit, which covers the whole of the area studied, has a policy of active case finding that further increases data quality. Moreover, as the findings do not change if the analyses are repeated using mortality data, it is unlikely that incomplete case ascertainment is a problem in this study.

The use of weighted averages of PCT deprivation scores can potentially hide areas of high or low deprivation within a PCT thus increasing ecological bias. However, this kind of analysis is not possible at ward level as the number of procedures would be too small to permit the calculation of meaningful rates. The use of PCTs provides suitable units of aggregation and the use of a weighted average IMD score is an appropriate way of including deprivation in the analysis.

An imbalance between need (as measured by mortality or disease incidence) and service provision (operation rates) has been described for other diseases, notably coronary heart disease. ${ }^{12}$ There has been little published evidence to suggest that provision of lung cancer surgery may not be equitable.
This study suggests that in the east of England access to lung cancer surgery at PCT level does not correlate well with need. Using control charts to analyse the study data permits both the quantification of inequity, and the identification of those parts of the healthcare system in which inequity is present. Control charts are quick and easy to produce and do not require a lot of complex calculation. The technique can also be applied to practice level data and so control charts could also be used within a single PCT.

\section{ACKNOWLEDGEMENTS}

The authors would like to thank Sara Godward and Leigh Roberts from the East Anglian Cancer Intelligence Unit for supplying lung cancer incidence data.

\section{Authors' affiliations}

J Battersby, J Flowers, Eastern Region Public Health Observatory, Institute of Public Health, Cambridge, UK

I Harvey, School of Medicine, Health Policy and Practice, University of East Anglia, Norwich, UK

Funding: none.

Competing interests: none declared.

\section{REFERENCES}

1 Department of Health. Improvement, expansion and reform: the next 3 years. London: Department of Health, 2003. http://www.doh.gov.uk/ planning2003-2006/improvementexpansionrefrom.pdf (accessed 23 May 2003).

2 Hamer L, Jacobson B, Flowers J, et al. Health equity audit made simple: a briefing for primary care trusts and local strategic partnerships. Cambridge: Association of Public Health Observatories, Health Development Agency (East), 2003.

3 Flowers J, Pencheon D. Introduction to health equity audit. Information on Public Health Observatory recommended methods. Cambridge: Eastern Region Public Health Observatory, 2002.

4 Quinn M, Babb P. Cancer trends in England and Wales, 1950-1999. Health Statistics Quarterly 2002;8:6-19.

5 NHS Centre for Reviews and Dissemination. Management of lung cancer. Effective Health Care Bulletin 1998;4:1-12.

6 Cartman ML, Hatfield AC, Muers MF, et al. Lung cancer: district active treatment rates affect survival. J Epidemiol Community Health 2002;56:424-9.

7 Department of Health. Guidance on commissioning cancer services: improving outcomes in lung cancer. London: Department of Health, 1998.

8 NHS Information Authority. Cancer data manual-lung cancer appendix version 2. http://www.nhsia.nhs.uk/cancer/pages/dataset/docs/ Lung_appendix.pdf (accessed 23 May 2003).

9 Regeneration Research. Indices of deprivation 2000. London: Department of the Environment, Transport and the Regions, 2000.

10 Mohammed MA, Cheng KK, Rouse A, et al. Bristol, Shipman, and clinical governance: Shewhart's forgotten lessons. Lancet 2001;357:463-7.

11 Mosteller F, Tukey J. The uses and usefulness of binomial probability paper. J Am Stat Assoc 1949;44:174-212.

12 Ben Shlomo Y, Chaturvedi N. Assessing equity in access to health care provision in the UK: does where you live affect your chances of getting a coronary artery bypass graft? J Epidemiol Community Health 1995;49:200-4. 